\title{
EDITORIAL
}

\section{Pasando de SER Un INVESTIGador ÉTICO A UN AUTOR ÉTICO}

La ética es relevante para cada fase de una investigación, incluyendo el desarrollo de la pregunta, diseño del protocolo de estudio, aprobación ética, reclutamiento de los participantes, análisis de datos y reporte de resultados. En esta última fase -el reporte de resultados- los investigadores pasan a ser autores al redactar resúmenes enviados a congresos y/o al publicar en revistas científicas. En esta etapa de diseminación del trabajo, los autores necesitan entender y aplicar estándares internacionales y pautas éticas de investigación. El propósito de este artículo es revisar la ética de publicación focalizando en la definición de autoría, la identificación de organizaciones que definen estándares internacionales y la discusión del rol de la ética, incluyendo los estándares recomendados para las revistas de investigación científica.

\section{Criterios para establecer la autoría}

Los estándares estipulados por el International Committee of Medical Journal Editors (ICMJE) han sido identificados por las revistas líderes en ciencias de la salud y revistas biomédicas como las mejores prácticas para la publicación, por lo que los autores deberían adherirse a ellos. La ICMJE recomienda que la autoría esté basada en estos cuatro criterios:

1. Contribuciones sustanciales a la concepción o diseño del trabajo; o la adquisición, análisis o interpretación de datos para este trabajo;

2. Elaboración de un borrador del trabajo o revisarlo de manera crítica por su importante contenido intelectual;

3. Aprobación final de la versión a ser publicada;

4. Acuerdo para rendir cuentas sobre todos los aspectos del trabajo, asegurando que las preguntas relacionadas a la precisión o integridad de cualquier parte de este son apropiadamente investigadas y resueltas.

Se puede acceder a estos criterios y otros estándares ICMJE (International Committee of Medical Journal Editors) en el siguiente enlace: http://icmje.org/recommendations/.

Además, la American Psychological Association (APA) publica estándares de conducta de investigación, incluyendo ética de publicación y autoría, consideradas prácticas esenciales para los investigadores en las ciencias sociales y conductuales. De acuerdo con APA, la autoría requiere "reconocimiento público por la contribución científica o profesional para el segmento de información diseminada". Los Principios Éticos y Código de Conducta APA (2010, Sección 8.12) ofrecen las tres pautas siguientes para determinar autoría:

1. Los autores asumen la responsabilidad y el crédito, incluyendo el de autoría, solo por el trabajo que hayan realizado o en el que ellos hayan contribuido sustancialmente.

2. La autoría principal y otros créditos de publicación reflejan con precisión las contribuciones científicas o profesionales de los individuos involucrados a pesar de su posición. La mera posesión de un puesto institucional, como por ejemplo jefe 
de departamento, no justifica el crédito de autoría. Las contribuciones menores a la investigación o a la redacción para publicación son apropiadamente reconocidas, como en notas a pie de página o en una declaración introductoria.

3. Excepto bajo circunstancias excepcionales, un estudiante es listado como autor principal en cualquier artículo de múltiples autores que esté substancialmente basado en el trabajo doctoral del estudiante. Los docentes asesores deben discutir sobre el crédito de publicación con los estudiantes tan pronto como sea posible y a través de todo el proceso de investigación y publicación, de manera apropiada.

Se puede acceder a estas pautas y otros estándares APA en el siguiente enlace: https://www. apa.org/research/responsible/.

\section{Pautas sobre contribución de autor}

Los criterios ICMJE, en conjunto con las normas APA, definen el proceso que los investigadores éticos utilizan para determinar la autoría de la publicación. En ese sentido, los investigadores DEBEN participar en el desarrollo y/o implementación del proyecto de investigación, así como en la redacción y/o revisión del manuscrito para publicar, para ser considerados autores. En este sentido, la siguiente referencia rápida puede ser usada para determinar la elegibilidad de un investigador para autoría.

\begin{tabular}{|c|c|c|c|}
\hline \multicolumn{4}{|c|}{ Guía para determinar la autoría: Los criterios básicos } \\
\hline \multicolumn{2}{|c|}{ Categoría 1: Tipo A o B (obligatorio) } & Categoría 2 (obligatorio) & Categoría 3 (opcional) \\
\hline \multicolumn{2}{|c|}{ INVESTIGACIÓN } & PUBLICACIÓN & CONSIDERACIONES \\
\hline Tipo A: UN criterio & Tipo B: DOS criterios & UN criterio & NINGÚN criterio \\
\hline $\begin{array}{l}\text { Desarrollo del con- } \\
\text { cepto }\end{array}$ & $\begin{array}{l}\text { Concepto revisado } \\
\text { o nuevo }\end{array}$ & $\begin{array}{l}\text { Redactar el borrador } \\
\text { del manuscrito }\end{array}$ & $\begin{array}{l}\text { Aseguramiento } \\
\text { del financiamiento }\end{array}$ \\
\hline Diseño del estudio & $\begin{array}{l}\text { Diseño revisado } \\
\text { o nuevo }\end{array}$ & $\begin{array}{l}\text { Redactar secciones } \\
\text { importantes }\end{array}$ & Experticia estadística \\
\hline $\begin{array}{l}\text { Recolección de } \\
\text { datos }\end{array}$ & $\begin{array}{l}\text { Datos reconfigurados } \\
\text { y/o reutilizados }\end{array}$ & $\begin{array}{l}\text { Revisión por parte } \\
\text { del mentor* }\end{array}$ & Apoyo Administrativo \\
\hline Análisis de datos & $\begin{array}{l}\text { Análisis revisado } \\
\quad \text { o nuevo }\end{array}$ & $\begin{array}{l}\text { Revisión crítica } \\
\text { sustancial** }\end{array}$ & Apoyo con materiales \\
\hline $\begin{array}{l}\text { Interpretación y/o } \\
\text { resultados }\end{array}$ & $\begin{array}{l}\text { Nueva interpretación } \\
\text { con resultados }\end{array}$ & $\begin{array}{l}\text { Revisión de un experto } \\
\text { tequerida*** }\end{array}$ & Asesoramiento técnico \\
\hline \multicolumn{4}{|c|}{$\begin{array}{l}\text { *Revisión del mentor (se requiere recomendaciones de manera formal, consultas verbales y comentarios escri } \\
\text { tos críticos sustanciales). }\end{array}$} \\
\hline \multicolumn{4}{|c|}{$\begin{array}{l}\text { kevisión crítica sustancial (se requiere una revisión desde moderada a una más completa, con una amplia } \\
\text { edición del texto) para incorporar conocimientos sustanciales y correcciones cruciales. }\end{array}$} \\
\hline \multicolumn{4}{|c|}{$\begin{array}{l}\text { *** Revisión de un experto (se requiere revisión conceptual y de contenido para revisar y reenviar, o aborda } \\
\text { comentarios complejos de los revisores o déficits metodológicos importantes de manuscritos rechazados). }\end{array}$} \\
\hline
\end{tabular}


En varias partes del mundo, los estudiantes de pregrado realizan un proyecto de investigación, mientras que los estudiantes de maestría completan una tesis, y los estudiantes de doctorado realizan lo que se conoce en inglés como "dissertation" (trabajo que es sustancialmente más comprehensivo que una tesis). En términos de la guía presentada previamente, todas las investigaciones, producidas por académicos o estudiantes investigadores, corresponden a proyectos de investigación. La sección específica de investigación (categoría 1), tipo A y tipo $\mathrm{B}$, tiene importancia para investigadores calificados y estudiantes de doctorado. Sin embargo, el tipo B tiene una importancia particular para los investigadores principiantes, investigadores no calificados y estudiantes de pregrado y maestría.

En el caso de un manuscrito que proviene de un proyecto de investigación original de un estudiante (Categoría 1A), este debería ser el primer autor (suele ser el caso de los estudiantes de doctorado). La EXCEPCIÓN corresponde al estudiante que no estuvo involucrado en la redacción de la mayoría de las partes del manuscrito. Se presume que este estudiante fue invitado, pero eligió no participar en la preparación del escrito. Así también, existen estudios de investigación secundaria (Categoría 1B), que requieren análisis secundario de datos; en algunos casos utilizando datos de la investigación original realizada por estudiantes (puede ser el caso de los estudiantes de pregrado o maestría). En este caso, los datos existentes de la investigación original se trasladan a un nuevo proyecto con una nueva pregunta de investigación usualmente con variables diferentes para el análisis de datos. Es importante destacar que los mentores y los asesores deben tratar de involucrar a los estudiantes en los proyectos que utilizan su recopilación de datos para el análisis secundario.

Hay tres puntos importantes y específicos para la investigación y publicación de estudiantes. Primero, es necesario terminar la tesis requerida por la universidad y participar en la redacción del manuscrito para su publicación. Segundo, los mentores y/o asesores NO tienen derecho a ser autores en el manuscrito a publicar a menos que cumplan con los criterios para ambos puntos: la investigación original (Categoría 1A) o el análisis secundario (Categoría 1B) y la posterior publicación (Categoría 2). En el caso del mentor y/o asesor supervisor de la investigación del estudiante, cualquier política universitaria o costumbre académica que contradiga esta norma constituye una conducta antiética que debería ser reportada inmediatamente a la revista y a la universidad o a la autoridad nacional de investigación responsable de la conducta ética de los investigadores, si el manuscrito fue publicado.

Finalmente, las personas involucradas de manera mínima en informar el estudio o redactar el manuscrito probablemente no serán elegibles para autoría. En el caso que la autoría no sea claramente definida con los criterios de las categorías 1 o 2, se debe mirar la categoría 3 (criterio opcional) para evaluar los roles adicionales de los investigadores, que pueden fundamentar la inclusión del investigador como autor. Esto provee cierta flexibilidad cuando existan dudas respecto a qué tan adecuadamente se cumple con dichos criterios.

Sin embargo, cuando un investigador no satisface los criterios de autoría, se le debe considerar para reconocimientos en la publicación. Esto incluye a las personas que brindaron asistencia técnica, revisaron los primeros borradores del manuscrito, proporcionaron sus servicios sin compensación (por ejemplo, contar con un estadístico fuera de la institución), y apoyaron el proyecto de alguna otra manera importante que 
se puede definir claramente. Como se mencionó anteriormente, cuando una persona es considerada pero no es elegible para autoría, probablemente sea apropiado nombrarla en la sección de reconocimientos.

\section{Tipos de autoría}

La posición más importante en una publicación es el primer autor. Este autor es usualmente el investigador que guía la investigación, y la posterior publicación para diseminar los resultados. Como tal, se considera que el primer autor ha contribuido con la mayor parte de la investigación. Por esta razón, los artículos de investigación con autores múltiples son a menudo citados como "Domínguez et al." mencionando al primer autor y a los otros autores, definidos por "et al."

Posiblemente el segundo autor más importante es el último autor. En este sentido, el último autor es generalmente el investigador sénior, especialista en el tema, y que es usualmente el mentor del estudio que terminará en manuscrito. A menudo, el último autor está involucrado en muchos otros pasos de la investigación, así también en los procesos de publicación. Este autor brinda a los investigadores la guía experta y conocimiento experiencial para mejorar la calidad general del trabajo. Su rol es esencial para hacer avanzar la investigación y el manuscrito hacia su publicación. Esta es la razón por la que en el estilo APA, cuando hay más de siete autores en la referencia, los primeros seis autores son seguidos por un punto, punto, punto, con todos los autores adicionales reflejados (por el punto, punto, punto) y luego el último autor. Aquí hay un ejemplo: Krederdt, S. L., Domínguez, K. A., Jiménez, R., Paz, M. Y., Mendigure, J., Leyva, J. M., ... Palmieri, P. A. (2019).

Finalmente, el autor corresponsal es usualmente la persona responsable de seleccionar la revista, comunicarse con el editor sobre su intención de enviar el manuscrito, preparándolo en la forma y estilo correctos, enviándolo a la revista, respondiendo preguntas de la revista durante el proceso de revisión, coordinando el trabajo de revisar y reenviar el manuscrito, y completando la documentación necesaria una vez que el manuscrito sea aceptado. Es importante mencionar que el autor corresponsal es habitualmente responsable de identificar una nueva revista para la presentación del manuscrito cuando este es rechazado.

\section{IV. Ética para publicación de investigación}

El Committee on Publication Ethics (COPE) brinda una guía sobre la ética en publicación, además de recursos para educar y apoyar a autores y editores con respecto a la adhesión a los estándares éticos de publicación. Este comité publica estándares de práctica para la transparencia en la evaluación individual sobre contribuciones de autor a manuscritos, y procesos claros para tratar potenciales disputas de autoría. Véase el siguiente enlace: https://publicationethics.org/.

En particular, el COPE promueve la integridad en la investigación y publicación académicas a través de diez prácticas centrales. Estas prácticas son aplicables a todas las personas que intervienen en la publicación en revistas académicas. De acuerdo al COPE, estas deberían ser consideradas como estándares para autores y revistas, sumados a los 
estándares nacionales e internacionales para investigadores. Asimismo, cada revista debe tener "prácticas robustas y bien descritas, así como documentadas públicamente" para las siguientes áreas:

1. Acusaciones de mala conducta: las revistas deben tener un proceso claramente descrito para manejar acusaciones, sin importar cómo fueron captados por la revista o editorial. Las revistas deben tomar seriamente las acusaciones de mala conducta pre- y pospublicación. Las políticas deberían incluir cómo manejar las acusaciones de los denunciantes.

2. Autoría y contribución: deberían instituirse políticas claras para requerimientos de autoría y contribución (que permitan la transparencia alrededor de quién contribuyó al trabajo y en qué capacidad), así como procesos para manejar potenciales disputas.

3. Quejas y apelaciones: las revistas deberían tener un proceso claramente descrito para tratar las quejas contra la revista, su personal, la editorial o su junta editorial.

4. Conflictos de interés/intereses en competencia: debe haber definiciones claras de conflictos de interés y procesos para manejar conflictos de intereses de autores, revisores, editores, revistas y editoriales, sean identificados antes o después de la publicación.

5. Datos y reproducibilidad: las revistas deben incluir políticas de disponibilidad de datos y alentar el uso de pautas de reporte y de registro de ensayos clínicos y otros diseños de estudio de acuerdo con la práctica estándar en su disciplina.

6. Supervisión ética: la supervisión ética debería incluir, pero no limitarse, a, políticas de consentimiento de publicación, publicaciones sobre poblaciones vulnerables, conducta ética de investigación en animales, conducta ética de investigación en seres humanos, manejando prácticas éticas de manejo de datos confidenciales, así como prácticas empresariales y comerciales éticas.

7. Propiedad intelectual: todas las políticas sobre propiedad intelectual, incluyendo copyright (derechos de autores) y licencias de publicación, deberían ser descritas claramente. Además, cualquier costo asociado a la publicación debería ser obvio para los autores y lectores. Las políticas deberían ser claras sobre lo que constituye la prepublicación que imposibilitará la consideración. Se debería ser específico sobre lo que constituye plagiarismo y publicación redundante/superpuesta.

8. Administración de la revista: una estructura bien descrita e implementada es esencial, incluyendo el modelo de negocio, políticas, procesos y software para el funcionamiento eficiente de una revista editorialmente independiente, así como el manejo y formación eficientes de las juntas editoriales, el personal de la editorial y de publicación.

9. Procesos de revisión de pares: todos los procesos de revisión de pares deben ser descritos de manera transparente y bien gestionados. Las revistas deberían brindar capacitación a editores y revisores y tener políticas en diversos aspectos de revisión de pares, especialmente con respecto a adopción de modelos apropiados de revisión y procesos para manejar conflictos de interés, apelaciones y disputas que podrían emerger en la revisión de pares.

10.Discusiones y correcciones pospublicación: las revistas deben permitir el debate pospublicación ya sea en su sitio web, a través de cartas al editor, o en un sitio moderado externo, como PubPeer. Estos deben tener mecanismos para corregir, revisar o retractar artículos después de ser publicados. 
Se puede acceder a estas pautas y otros estándares CORE (Committee on Publication Ethics) en el siguiente enlace: https://publicationethics.org/core-practices.

\section{Conclusión}

En términos de conducta ética en la publicación, se requieren lineamientos claros y concisos en la definición de los criterios básicos para autoría y el establecimiento del orden adecuado de los autores, puntos que esta editorial ha intentado presentar. Juntos, la APA (American Psychological Association), el ICMJE (International Committee of Medical Journal Editors), y el COPE (Committee on Publication Ethics) brindan las mejores prácticas para los investigadores dedicados a esta, en conjunto con las guías éticas aceptadas para los autores en sus publicaciones. Los investigadores siempre son responsables de aprender, comprender y aplicar estas prácticas y guías durante su investigación, así como cuando publican sus resultados. Finalmente, los mentores y sus universidades, los editores y sus revistas son responsables de la adhesión a estas prácticas. De esta forma, todos somos responsables de mantener un ambiente ético de publicación.

PATRICK A. PALMIERI

Vicerrectorado de Investigación Universidad Norbert Wiener 